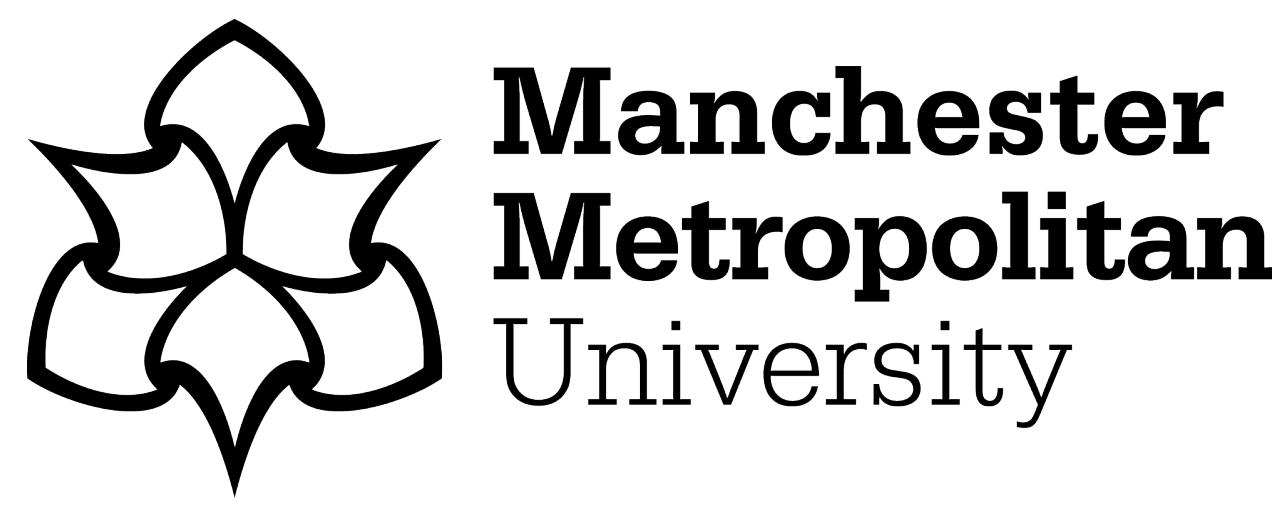

Dorado-Correa, Adriana M, Zollinger, Sue Anne ORCID logoORCID: https://orcid.org/0000-0001-8819-2606 and Brumm, Henrik (2017) Vocal plasticity in mallards: multiple signal changes in noise and the evolution of the Lombard effect in birds. Journal of Avian Biology, 49 (1). ISSN 0908-8857

Downloaded from: https://e-space.mmu.ac.uk/623442/

Version: Accepted Version

Publisher: Wiley

DOI: https://doi.org/10.1111/jav.01564

Usage rights: Creative Commons: Attribution 4.0

Please cite the published version 


\title{
Vocal plasticity in mallards: multiple signal changes in noise and the evolution of the Lombard effect in birds
}

\author{
Adriana M. Dorado-Correa, Sue Anne Zollinger, Henrik Brumm
}

Max Planck Institute for Ornithology, Communication and Social Behaviour Group, Eberhard-GwinnerStr., 82319 Seewiesen, Germany

Corresponding author: Henrik Brumm, Max Planck Institute for Ornithology, Communication and Social Behaviour Group, Eberhard-Gwinner-Str., 82319 Seewiesen, Germany. Email: brumm@orn.mpg.de

Decision date: $02-$ Oct-2017

This article has been accepted for publication and undergone full peer review but has not been through the copyediting, typesetting, pagination and proofreading process, which may lead to differences between this version and the Version of Record. Please cite this article as doi: [10.1111/jav.01564]. 


\section{Abstract}

Signal plasticity is a building block of complex animal communication systems. A particular form of signal plasticity is the Lombard effect, in which a signaler increases its vocal amplitude in response to an increase in the background noise. The Lombard effect is a basic mechanism for communication in noise that is well-studied in human speech and which has also been reported in other mammals and several bird species. Sometimes, but not always, the Lombard effect is accompanied by additional changes in signal parameters. However, the evolution of the Lombard effect and other related vocal adjustments in birds are still unclear because so far only three major avian clades have been studied. We report the first evidence for the Lombard effect in an anseriform bird, the mallard (Anas platyrhynchos). In association with the Lombard effect, the fifteen ducklings in our experiment also increased the peak frequency of their calls in noise. However, they did not change the duration of call syllables or their call rates as has been found in other bird species. Our findings support the notion that all extant birds use the Lombard effect to solve the common problem of maintaining communication in noise, i.e. it is an ancestral trait shared among all living avian taxa, which means that it has evolved more than 70 million years ago within that group. At the same time, our data suggest that parameter changes associated with the Lombard effect follow more complex patterns, with marked differences between taxa, some of which might be related to proximate constraints.

Keywords: Anas platyrhynchos, animal communication, Lombard effect, noise, signal plasticity 


\section{Introduction}

Signal plasticity is a key feature of derived animal communication systems that allows individual signal adjustments in response to changes in the environment. Animals use this capacity in situations such as changing social contexts (e.g. Janik 2000, Brumm and Slater 2006a, Gavassa et al. 2013) or coping with challenging conditions for signal detection in fluctuating environments (e.g. Lengagne et al. 1999, Ord et al. 2007, Goodwin and Podos 2013). Indeed, there is growing evidence that signal detection constraints are one of the major forces driving the evolution of animal communication systems across different taxa (Brumm 2013, Wiley 2015).

In acoustic communication, signal detection is particularly constrained by background noise (Klump 1996, Brumm and Slabbekoorn 2005). One efficient form of signal plasticity that mitigates masking by noise is the Lombard effect, i.e. the regulation of vocal amplitude in relation to the noise level (Zollinger and Brumm 2011). This phenomenon is found in many birds and mammals, including human speech (reviewed in Brumm and Zollinger 2011, Hotchkin and Parks 2013). Recently, the Lombard effect has also been reported in two frog species (Halfwerk et al. 2016, Shen and Xu 2016) but it is absent in another anuran species (Love and Bee 2010), and it is also absent in a non-avian reptile (Brumm and Zollinger 2017).

Several studies have addressed how the strength of the Lombard effect is affected by noise characteristics (e.g. Sinnott et al. 1975, Manabe et al. 1998, Brumm and Todt 2002, Stowe and Golob 2013) or social factors (e.g. Kobayasi and Okanoya 2003, Garnier et al. 2010). Others have investigated the neural substrate underlying the effect (Nonaka et al. 1997, Hage et al. 2006, Eliades and Wang 2012). In echolocating bats and singing canaries (Serinus canaria), the Lombard effect is triggered at least 30320 milliseconds after noise onset (Hage et al. 2013, Hardman et al. 2017, Luo et al. 2017a), which means that vocal amplitude can track noise levels more or less in real time.

Sometimes the Lombard effect is accompanied by changes in other vocal parameters, although such multiple adjustments are not universal (reviewed in Brumm and Zollinger 2011). For instance, primates (e.g. Van Summers et al. 1988, Brumm et al. 2004, Hotchkin et al. 2015), a bat (Luo et al. 2015), a songbird (Leonard and Horn 2005), and a parrot (Osmanski and Dooling 2009) also increase the duration of brief vocalizations, which further increases detectability (Klump and Maier 1990, Pohl et al. 2013, Luo et al. 2015). In contrast, domestic fowl (Gallus gallus) do not increase call syllables duration with Lombard-induced increases in amplitude (although, like other birds, they show the Lombard effect) (Brumm et al. 2009). Likewise, spectral signal changes may also accompany the Lombard effect in some 
species but not in others. In human speech, for instance, the Lombard effect is often associated with a concurrent increase in fundamental frequency and/or a shift in spectral tilt (e.g. Lienard and Di Benedetto 1999, Jessen et al. 2005, Cooke and Lu 2010). Both phenomena have also been found in the calls of a New World monkey (Hotchkin et al. 2015). In contrast, echolocating bats may adjust their call frequencies in noise but this response appears to be independent from the Lombard effect (e.g. Hage et al. 2014, Luo \& Wiegrebe 2016). In birds, Lombard-related increases in vocal pitch were observed in a tinamou (Schuster et al. 2012) and a parrot (Osmanski and Dooling 2009) but not in songbirds (Potvin and Mulder 2013, Templeton et al. 2016, Zollinger et al. 2017). These differences between bird species cannot be explained by the fact that songbirds are vocal learners because parrots also acquire their calls through vocal production learning. It may be that the concurrent increase of amplitude and pitch in some species reflects a physical coupling of the two parameters during vocal production (Beckers et al. 2003, Elemans et al. 2008) that can be decoupled in songbirds. The fact that the Lombard effect is widespread in birds and mammals, while the suite of associated parameter changes differs between species, parallels findings from modelling studies suggesting that the Lombard effect is more efficient in increasing signal detection in noise than the other frequently observed changes in other signal parameters (Nemeth and Brumm 2010, Luo et al. 2015).

Despite the wealth of studies on the Lombard effect in many different species, the evolutionary history of the trait remains unclear. Though the effect is present in all tested bird and mammal species (reviewed in Brumm and Zollinger 2013, Hotchkin and Parks 2013) it is not possible to establish whether or not it is ancestral in either clade because in both groups major lineages have not yet been investigated. Extant birds are divided into Palaeognathae, and Neognathae, with the latter comprising more than $99 \%$ of all extant species (Mayr 2017). The Lombard effect is present in a paleognath clade, the Tinamiformes, and in two of the three neognath lineages, the Galliformes and Neoaves (reviewed in Brumm and Zollinger 2011). However, the character state is not documented in the Anseriformes, the third neognath lineage. To fill this gap, we tested whether an anseriform bird, the mallard (Anas platyrhynchos), exhibits the Lombard effect. We also examined if a potential Lombard effect is accompanied by other call parameter changes in this species. The results will help reconstructing the phylogenetic origin of the Lombard effect and other related vocal changes in birds and they will also allow testing the hypothesis that an uncoupling of amplitude and pitch during the Lombard effect is a derived feature of songbirds.

'This article is protected by copyright. All rights reserved.' 


\section{Methods}

Animals and housing

The experiment was conducted at the Max Planck Institute for Ornithology in Radolfzell, Germany. We used 15 mallard ducklings, aged between two to ten days post hatching. The birds came from three different clutches and all subjects were kept with their parents and siblings in family outdoor aviaries $(3.95 \times 2.90 \times 2.60 \mathrm{~m})$, equipped with a pond and roof. The birds were fed on an ad libitum diet.

As in many precocial birds, vocalizing in mallard ducklings can readily be elicited by separating them from their brood (Gaioni and Evans 1986, Gaioni and Platte 1982). Typical mallard separation calls consist of series of several of relatively short, frequency modulated call elements (Fig. 1).

\section{Experimental set-up}

The subjects were placed singly in a wire cage $(48 \times 36 \times 60 \mathrm{~cm})$ in the centre of a test arena $(2.55 \times 1.64 \mathrm{~m})$ which was surrounded by sound absorbing materials. An omnidirectional microphone (Sennheiser ME66) connected to a digital recorder (Marantz PMD660) was suspended $40 \mathrm{~cm}$ above the cage to record the calls of the ducklings. Recording with the microphone above the animal and facing downwards reduces variation due to the orientation of the animal and the directional pattern of vocal sound radiation (Brumm 2002, Brumm and Zollinger 2011). Filtered white noise (bandwidth 0-12 kHz) was broadcast from a PC, fed through an amplifier (Technics SU-V300M2) and then to four loudspeakers (JBL PRO III N108) that were placed in the middle of each of the four sides of the arena. Experimental treatments consisted of noise played at two levels, 60 and $70 \mathrm{~dB}(\mathrm{~A})$ SPL (measured with a Voltcraft SL 400 sound level meter in the center of the test cage at the height of the ducklings' heads) and no noise in random order. The mean ambient noise level, when no experimental noise was broadcast, was $44 \mathrm{~dB}(\mathrm{~A})$ SPL. After a duckling had produced at least 15 call elements, which took on average about one minute, the noise treatment was changed.

\section{Acoustic analysis and statistics}

The calls were recorded as WAV files with a sampling rate of $44.1 \mathrm{kHz}$ and 16-bit resolution. All acoustic analyses were done using the software using Avisoft SASLab Pro version 5.2.0.8 (Avisoft Bioacoustics, Germany). Call rates were determined from spectrograms (FFT size 1024 points, Hamming window) and the peak frequencies and durations of the call elements were measured using the automatic 
measurement function of Avisoft (durations were measured at $10 \mathrm{~dB}$ below the peak, frequency resolution: $43.1 \mathrm{~Hz}$ ). The maximum rms amplitude of each element was measured with an integration time of 50 milliseconds and then the background noise value was subtracted following published procedures (Brumm and Zollinger 2011). The amplitude measurements were calibrated with a recording of white noise of known sound pressure level recorded with the experimental recording set-up (70 dB SPL, measured at the position of the recording microphone with a Voltcraft SL 400 SPL meter).

All statistical analyses were performed with R 3.1.1 (R Core Team 2013). We fitted linear mixed-effects models and general linear models using the "lmer" and "glmer" functions (R package lme4). Additionally, we used the "sim" function (package arm) to simulate the posterior distribution of the model parameters, values were extracted based on 2000 simulations (Gelman and Hill 2007). The statistical significance of fixed effects and interactions were assessed based on the $95 \%$ credible intervals (CI) around the mean (estimate). We considered an effect to be "significant" in the frequentist's sense when the 95\% CI did not overlap zero. We fitted one model for each call parameter, our response variables were the parameters and background noise (dB SPL) was fitted as fixed effect and individual ID as random effect. To examine effects of noise on call rate and the number of elements per series we fitted general linear models $(\mathrm{glm})$ with a poisson distribution. In the call-rate model we fitted the number of calls as the response variable, taking into account in the model duration of the series as a covariate. Since all the individuals were tested with the three treatments, all the models were run taking into account the order in which the treatment were presented to each individual, as well as the age of the individual (dayspost hatched). However we did not find any significant correlations with the order of treatment nor with age. Thus, we present only the reduced models without these variables. A general correlation between the peak frequency and the amplitude of call elements was investigated with a Spearman rank correlation. For this test, we randomly selected 45 elements from each individual, irrespective of the noise treatment (for five birds 45 elements was the total number of elements recorded during the experiment).

\section{Results}

On average we recorded 3 call series comprising in total between 15 and 30 elements per bird and treatment (median: 17 elements). The amplitude of the call elements increased significantly in response to an increase of background noise (Table 1, Fig 2). Thus, the subjects tested showed the Lombard effect, with an average increase of a $4.8 \mathrm{~dB}$ between no-noise (when ambient noise was approx. $44 \mathrm{~dB}(\mathrm{~A}) \mathrm{SPL}$ ) 
and the $70 \mathrm{~dB}$ noise conditions. The 10-day old birds showed a much stronger Lombard effect than the younger birds, with individual increases of up to over $20 \mathrm{~dB}$ (Fig. 3).

In addition to the Lombard effect, the ducklings also increased the peak frequency of their call elements in noise (Table 1, Fig. 2). Between the no-noise and the $70 \mathrm{~dB}$ conditions call peak frequencies increased on average by $227 \mathrm{~Hz}$. Peak frequency and call level were significantly correlated independent of the noise treatment (Pearson correlation: $\mathrm{r}=0.44 ; \mathrm{N}=675, \mathrm{p}<0.001 ;$ Fig. 4).

In contrast, increases in noise level resulted in no significant changes in call element duration, call rate or serial redundancy, measured as the number of elements per call series (Table 1, Fig. 2).

\section{Discussion}

We found that mallards exhibited the Lombard effect and that this noise-dependent regulation of call amplitude was associated with an increase in call peak frequencies. In contrast, the tested birds did not adjust their duration of call elements, their call rates or the serial redundancy of call series. The presence of the Lombard effect in an anseriform bird corroborates the notion that the common problem of communicating in noisy environments has led to the common solution of the Lombard effect in birds (Brumm and Zollinger 2013).

\section{Multiple signal changes in noise}

Concurrent with the Lombard effect, the ducklings also increased the peak frequency of their call elements. Such additional parameter changes are often associated with the Lombard effect, but which parameters exactly are adjusted differs between species and contexts (reviewed in Brumm and Zollinger 2011). In some cases, a whole suite of spectral and temporal traits changes along with the Lombard effect, e.g. in human speech, but not every signal change is necessarily adaptive in the context of signal transmission (Brumm and Zollinger 2011). For instance, a rise in vocal pitch, like the one that we observed in our ducklings, typically occurs during the Lombard effect irrespective of any release from signal masking (Osmanski and Dooling 2009, Lu and Cooke 2009, Schuster et al. 2012).

Like tinamous (Schuster et al. 2012) and parrots (Osmanski and Dooling 2009), but unlike songbirds (Potvin and Mulder 2013, Templeton et al. 2016, Zollinger et al. under revision), the Lombardinduced increase in call amplitude led to a concurrent increase in vocal pitch in the ducklings in our study. This finding supports the hypothesis that a decoupling of amplitude and frequency during the Lombard 
effect may be a derived trait of songbirds. The increase in call frequency in noise in ducks and other birds is most likely a passive by-product of the Lombard effect, due to a physical coupling of amplitude and frequency during phonation (Beckers et al. 2003, Elemans et al. 2008). Perhaps the more derived syrinx anatomy of songbirds (King 1989) allows them to control amplitude and frequency of their vocalizations independently and thus enables them to uncouple both parameters during Lombard-induced increases of vocal amplitude. Such capacity might be related to the sophisticated fine-tuning of air pressure by the songbird syrinx that controls the modulation of fundamental frequency (Amador and Margoliash 2013).

The lack of noise-dependent adjustments of call element durations in our ducks is in line with previous findings in domestic fowl (Brumm et al. 2009). However, birds more distantly related to ducks, such as parrots (Osmanski and Dooling 2009), as well as primates (e.g. Van Summers et al. 1988, Brumm et al. 2004, Hotchkin et al. 2015) and bats (Luo et al. 2015) do increase call element durations in Lombard-induced vocalizations and it remains to be investigated why domestic fowl and mallards do not. The absence of this trait deserves particular attention because an increase in element duration increases the detectability of the calls (Klump and Maier 1990, Pohl et al. 2013, Luo et al. 2015) and thus helps maintain communication in constant broad-band noise as it was used in our and other experiments. Both ducks and domestic fowl belong to an early divergent bird clade, the Galloanserae, and the lack of duration adjustments in noise might be indicative that this capacity is a synapomorphy of Neoaves (and a convergent trait in mammals).

We also did not find evidence for noise-related adjustments of call rate nor serial redundancy in the tested ducklings. This is in contrast to previous studies on galliform birds (the sister taxon of the Anseriformes): Japanese quail (Coturnix coturnix japonica) increased both their call rates and the number of elements per call series in response to experimentally elevated noise levels (Potash 1972). Domestic fowl also increase their call rates along with increasing noise levels but at noise amplitudes above $70 \mathrm{~dB}$ they decrease call rates again (Brumm et al. 2009). Further evidence for a noise-related adjustment of serial signal redundancy comes from correlative field studies in other neoaves: as the environmental noise levels rises, king penguins (Aptenodytes patagonicus) produce more call syllables (Lengagne et al. 1999) and chaffinches (Fringilla coelebs) repeat song types more often (Brumm and Slater 2006b). Taken together, the evidence shows that birds do not exercise an increase in call rates or redundancy for communication in noise as generically as the Lombard effect. Maybe the separation of the ducklings in our experiment already triggered maximum call rates and thus the tested birds could not further increase their call rates in noise. Considering that the variation in call rates did not differ between individuals, this 
might well be the case. Alternatively, the lack of increased call rates in noise may be due to the fact that some species utilize call element rates to encode information (Bradbury and Vehrencamp 2011) and therefore rates cannot be adjusted to increase signal transmission without compromising the information content of the signal.

Interestingly, some of the changes that may be associated with the Lombard effect can also occur in species that do not exhibit a Lombard effect. For example, Cope's grey tree frogs (Hyla chrysoscelis) increase their call duration in masking noise, even though call amplitude does not change (Love and Bee 2011). Likewise, tokay geckos (Gekko gecko) do not exhibit the Lombard effect, but, like the tree frogs, do increase the duration of their call syllables in noise (Brumm and Zollinger 2017). These findings demonstrate that some noise-related signal changes may occur independently from the Lombard effect and are perhaps phylogentically older than it.

\section{Ontogeny of the Lombard effect}

Although we did not design our experiment to investigate the ontogeny of the Lombard effect in mallards, our observation that it is already present in birds as young as 2 days after hatching has implications for the development of this vocal trait. Previous studies have also documented the Lombard effect in young animals: in domestic chicken at four weeks after hatching (Brumm et al. 2009), in nestling tree swallows (Tachycineta bicolor) nine to ten days posthatch (Leonard and Horn 2005), and in in pale spear-nosed bats (Phyllostomus discolor) two weeks after birth (Luo et al. 2017b). These and our findings demonstrate an early developmental origin of the Lombard effect, suggesting a fast maturation of the neural circuits responsible for auditory-vocal feedback in birds and mammals. The neural circuits underlying the Lombard effect are thought to be located in the brainstem (Nonaka et al. 1997, Hage et al. 2006) but higher cortical areas may also be involved (Eliades and Wang 2012). The early ontogenetic emergence of the effect suggests rather basic brain mechanisms that do not require extended maturation after birth. This basic control of the Lombard effect may then be modified by higher-level processes that may fully develop later in life. In addition, the magnitude of the Lombard effect will also be affected by the general development of the vocal apparatus, and thus may increase when animals increase their vocal power capabilities during ontogeny (Brumm and Hultsch 2001, Leonard and Horn 2006). Such a developmental change may explain why the magnitude of the Lombard effect in young animals increased with age (Luo et al. 2017b) because older and larger animals may be able to produce louder vocalizations and therefore can use a wider range of amplitudes. It could also be that the vocal motor control of amplitude improves 
with age. The latter may be accounted for our observation that ten-day old ducklings showed a much stronger Lombard effect than the younger birds not because the older birds produced higher call amplitudes in noise but rather they produced lower ones in the control treatment, suggesting greater control over amplitude modulation rather than increased power capabilities. However, the three ten-day old individuals in our experiment were all from the same clutch and thus we cannot exclude potential, yet unknown, genetic effects.

\section{Evolution of the Lombard effect}

Our results are in line with the hypothesis that the Lombard effect is present in all extant birds (Brumm and Zollinger 2011). With the new data on mallards the Lombard effect is now documented for all three major lineages of the Neognathae (Fig. 5). Therefore, we may conclude that the trait is probably ancestral in Neognathae, which comprise more than $99 \%$ of all extant bird species. The remaining species belong to an early diverging clade, the Palaeognathae, in which the Lombard effect is present in Tinamiformis (Fig. 5). If the effect is also a shared trait of the Palaeognathae, then it is most likely a synapomorphy of all birds. To test this hypothesis, the character state needs to be documented in other paleognathous species, especially ostriches and rheas, because the presence of the Lombard effect in these groups would strongly suggest that the trait was present in the common ancestor of the Palaeognathae (Fig. 5).

Presuming that the Lombard effect is ancestral in birds, we can proceed to consider broader patterns of its evolutionary history. The absence of the Lombard effect in a lizard (Brumm and Zollinger 2017) suggests that the noise-dependent regulation of vocal amplitude is either a convergent trait in birds and mammals or that it is a plesiomorphy that has been lost in Lepidosauria. Further Lombard experiments in Testudines and Crocodilia would be a critical test of these two alternative hypotheses. Both tortoises and crocodiles vocalize (Colafrancesco and Gridi-Papp 2016) and if, like geckos, they do not regulate their vocal amplitudes in relation to noise, it is likely that the Lombard effect has evolved independently in birds and mammals.

Published Lombard tests in frogs are still scarce and the overall picture remains unclear. Tungara frogs, Engystomops pustulosus, (Halfwerk et al. 2016) and concave-eared torrent frogs, Odorrana tormota, (Shen and Xu 2016) have been reported to adjust their call levels depending on the level of background noise. However, the Lombard effect is absent in Cope's grey tree frogs, Hyla chrysoscelis, (Love and Bee 2010). Thus the evolutionary history of the Lombard effect in frogs is still largely unresolved. In birds and mammals, the number of species for which the character state has been 
documented is also low, but all evidence supports the hypothesis of the Lombard effect being ancestral in both clades, whereas the current evidence in frogs does not. Some frogs may not show the Lombard effect because they always call close to their physical limitations and thus have little room for amplitude adjustments (Schwartz and Bee 2013). However, this is not true of all anuran species, as it has been shown that white-lipped frogs (Leptodactylus albilabris) can increase call amplitude in response to social cues (Lopez et al. 1988). Interestingly, males of this species may advertise in more rarefied choruses and Schwartz and Bee (2013) suggest that differences in the intensity of selection for maximizing call energy may be accounted for the differences in the ability to adjust call amplitude in anurans. On the other hand, the occurrence of the Lombard effect in birds and mammals suggests that either these animals are under less intense selection to maximize signal transmission or that the evolution of motor control over vocal amplitude enabled them to trade off the costs and benefits of high-amplitude signals (Zollinger and Brumm 2015).

\section{Conclusions}

Our study confirms an early appearance of the Lombard effect in terms of evolutionary history and ontogeny. The presence of the Lombard effect in an anseriform bird is in line with the hypothesis that the effect is a shared trait of all extant birds. If the Lombard effect is ancestral in birds, then, according to the phylogeny of Prum et al. (2015), it has evolved at least 70 million years ago. In addition to it being likely a shared character in birds and, probably convergently, in mammals, the Lombard effect in both groups appears to already be present in very young individuals, a few days after hatching or birth, respectively. These findings highlight how important the maintenance of vocal communication in changing environments is, and hence that the need to overcome noise should be regarded as a powerful driver of the evolution of animal communication systems.

\section{Acknowledgements}

We thank Wolfgang Fiedler and the animal care takers in Radolfzell for their support during the study. We also thank two anonymous reviewers for their comments on the manuscript. Funding was provided by the KAAD and the International Max Planck Research School for Organismal Biology (personal 
fellowships to A.M.D.C), the German Research Foundation (awards Br 2309/8-1 and Br 2309/8-2 to H.B.), and the Max Planck Society. 


\section{References}

Amador, A. and Margoliash, D. 2013. A Mechanism for Frequency Modulation in Songbirds Shared with Humans. - J. Neurosci. 33: 11136-11144.

Beckers, G. J. L., Suthers, R. A. and Ten Cate, C. 2003. Mechanisms of frequency and amplitude modulation in ring dove song. - J. Exp. Biol. 206: 1833-1843.

Bradbury, J. W. and Vehrencamp, S. L. 2011. Principles of animal communication, Sunderland, Massachusetts, Sinauer Associates.

Brumm, H. 2002. Sound radiation patterns in Nightingale (Luscinia megarhynchos) songs. - J. Orn. 143: 468-471.

Brumm, H. 2013. Animal Communication and Noise, Heidelberg Berlin, Springer-Verlag.

Brumm, H. and Hultsch, H. 2001. Pattern amplitude is related to pattern imitation during the song development of nightingales. - Anim. Behav. 61: 747-754.

Brumm, H., Schmidt, R. and Schrader, L. 2009. Noise-dependent vocal plasticity in domestic fowl. Anim. Behav. 78: 741-746.

Brumm, H. and Slabbekoorn, H. 2005. Acoustic communication in noise. - Adv. Stud. Behav. 35: 151209.

Brumm, H. and Slater, P. J. B. 2006a. Animals can vary signal amplitude with receiver distance: evidence from zebra finch song. - Anim. Behav. 71: 699-705.

Brumm, H. and Slater, P. J. B. 2006b. Ambient noise, motor fatigue and serial redundancy in chaffinch song. - Behav. Ecol. Sociobiol. 60: 475-481.

Brumm, H. and Todt, D. 2002. Noise-dependent song amplitude regulation in a territorial songbird. Anim. Behav. 63: 891-897.

Brumm, H., Voss, K., Köllmer, I. and Todt, D. 2004. Acoustic communication in noise: regulation of call characteristics in a New World monkey. - J. Exp. Biol. 207: 443-448.

Brumm, H. and Zollinger, S. A. 2011. The evolution of the Lombard effect: 100 years of psychoacoustic research. - Behaviour., 148: 1173-1198.

Brumm, H. and Zollinger, S. A. 2013. Avian vocal production in noise. In: Brumm, H. (ed.) Animal Communication and Noise. Springer-Verlag, Berlin Heidelberg, pp. 187-227.

Brumm, H. and Zollinger, S. A. 2017. Vocal plasticity in a reptile. - Proc. R. Soc. B 284: 20170451.

Colafrancesco, K. C. and Gridi-Papp, M. 2016. Vocal sound production and acoustic communication in amphibians and reptiles. In: Suthers, R. A. et al. (eds.) Vertebrate Sound Production and Acoustic Communication, Springer Handbook of Auditory Research 53, Springer Publishing Switzerland, pp. 5182.

Cooke, M. and Lu, Y. Y. 2010. Spectral and temporal changes to speech produced in the presence of energetic and informational maskers. - J. Acoust. Soc. Am. 128: 2059-2069.

Cynx, J., Lewis, R., Tavel, B. and Tse, H. 1998. Amplitude regulation of vocalizations in noise by a songbird, Taeniopygia guttata. - Anim. Behav. 56: 107-113.

Elemans, C. P. H., Zaccarelli, R. and Herzel, H. 2008. Biomechanics and control of vocalization in a nonsongbird. - J. R. Soc. Interface 5: 691-703.

Eliades, S. J. and Wang, X. Q. 2012. Neural correlates of the lombard effect in primate auditory cortex. J. Neurosci. 32: 10737-10748.

Gaioni, S. J. and Evans, C. S. 1986. Perception of distress calls in mallard ducklings (Anas platyrhynchos). - Behaviour 99: 250-274.

Gaioni, S. J. and Platte, P. L. 1982. Sibling interactions in mallard ducklings (Anas platyrhynchos). Behav. Analysis Lett. 2: 189-196.

Garnier, M., Henric, N. and Dubois, D. 2010. Influence of sound immersion and communicative interaction on the Lombard effect. - J. Speech Lang. Hear. Res .53: 588-608.

Gavassa, S., Roach, J. P. and Stoddard, P. K. 2013. Social regulation of electric signal plasticity in male Brachyhypopomus gauderio. - J. Comp. Physiol. A 199: 375-384.

Gelman; A. and Hill, J. 2007. Data analysis using regression and multilevel/hierarchical models. Cambrige University Press, New York.

Goodwin, S. E. and Podos, J. 2013. Shift of song frequencies in response to masking tones. - Anim. Behav. 85: 435-440.

Hage, S. R., Jiang, T. L., Berquist, S. W., Feng, J. and Metzner, W. 2014. Ambient noise causes independent changes in distinct spectro-temporal features of echolocation calls in horseshoe bats. $-J$. Exp. Biol. 217: 2440-2444.

Hage, S. R., Jiang, T. L., Berquist, S. W., Feng, J. and Metzner, W. 2013. Ambient noise induces independent shifts in call frequency and amplitude within the Lombard effect in echolocating bats. Proc. Nat. Acad. Sci. USA 110: 4063-4068. 
Hage, S. R., Jürgens, U. and Ehret, G. 2006. Audio-vocal interaction in the pontine brainstem during selfinitiated vocalization in the squirrel monkey. - Europ. J. Neurosci. 23: 3297-3308.

Halfwerk, W., Lea, A. M., Guerra, M. A., Page, R. A. and Ryan, M. J. 2016. Vocal responses to noise reveal the presence of the Lombard effect in a frog. - Behav. Ecol. 27: 669-676.

Hardman, S. I., Zollinger, S. A., Koselj, K., Leitner, S., Marshall, R. C. and Brumm, H. 2017. Lombard effect onset times reveal the speed of vocal plasticity in a songbird. - J. Exp. Biol. 220: 1065-1071.

Hotchkin, C. and Parks, S. 2013. The Lombard effect and other noise-induced vocal modifications: insight from mammalian communication systems. - Biol. Rev. 88: 809-824.

Hotchkin, C. F., Parks, S. E. and Weiss, D. J. 2015. Noise-induced frequency modifications of tamarin vocalizations: implications for noise compensation in nonhuman primates. - Plos One, $\mathbf{1 0 .}$

Janik, V. M. 2000. Whistle-matching in wild bottlenose dolphins (Tursiops truncatus). -Science, 289: 1355-1357.

Jessen, M., Löster, O. and Gfroerer, S. 2005. Influence of vocal effort on average and variability of fundamental frequency. - Int. J. Speech Lang. La., 12: 174-213.

King, A. S. 1989. Functional anatomy of the syrinx. . In: King, A. S. and McLelleand, J. (eds.) Form and Function in Birds. Academic Press, , London, pp. 105-220.

Klump, G. M. 1996. Bird communication in the noisy world. In Kroodsma, D. E. and Miller, E. H. (eds.) Ecology and Evolution of Acoustic Communication in Birds. Cornell University Press, Ithaca, pp. 321338.

Klump, G. M. and Maier, E. H. 1990. Temporal summation in the European starling (Sturnus vulgaris). J. Comp. Psychol. 104: 94-100.

Kobayasi, K. and Okanoya, K. 2003. Context-dependent song amplitude control in Bengalese finches. Neuroreport 14: 521-524.

Lengagne, T., Aubin, T., Lauga, J. and Jouventin, P. 1999. How do king penguins (Aptenodytes patagonicus) apply the mathematical theory of information to communicate in windy conditions? - Proc. R. Soc. B 26: 1623-1628.

Leonard, M. L. and Horn, A. G. 2005. Ambient noise and the design of begging signals. -Proc. R. Soc. B 272: 651-656.

Leonard, M. L. and Horn, A. G. 2006. Age-related changes in signalling of need by nestling tree swallows (Tachycineta bicolor). - Ethology, 112: 1020-1026.

Lienard, J. S. and Di Benedetto, M. G. 1999. Effect of vocal effort on spectral properties of vowels. $-J$. Acoust. Soc. Am. 106: 411-422.

Lopez, P. T., Narins, P. M., Lewis, E. R. and Moore, S. W. 1988. Acoustically induced call modification in the white-lipped frog, Leptodactylus albilabris. - Anim. Behav. 36: 1295-1308.

Love, E. K. and Bee, M. A. 2010. An experimental test of noise-dependent voice amplitude regulation in Cope's grey treefrog, Hyla chrysoscelis. - Anim. Behav. 80: 509-515.

Lu, Y. and Cooke, M. 2009. Speech production modifications produced in the presence of low-pass and high-pass filtered noise. - J. Acoust. Soc. Am. 126: 1495-1499.

Luo, J. and Wiegrebe, L. 2016. Biomechanical control of vocal plasticity in an echolocating bat. - J. Exp. Biol 219: 878-886.

Luo, J., Goerlitz, H. R., Brumm, H. and Wiegrebe, L. 2015. Linking the sender to the receiver: vocal adjustments by bats to maintain signal detection in noise. - Sci. Rep. 5: 18556.

Luo, J., Kothari, N. B. and Moss, C. F. 2017a. Sensorimotor integration on a rapid time scale. - Proc.Nat. Acad. Sci. USA 114: 6605-6610.

Luo, J. H., Lingner, A., Firzlaff, U. and Wiegrebe, L. 2017b. The Lombard effect emerges early in young bats: implications for the development of audio-vocal integration. - J. Exp. Biol. 220: 1032-1037.

Manabe, K., Sadr, E. I. and Dooling, R. J. 1998. Control of vocal intensity in budgerigars (Melopsittacus undulatus): Differential reinforcement of vocal intensity and the Lombard effect. - J. Acoust. Soc. Am. 103: 1190-1198.

Mayr, G. 2017. Avian Evolution: the Fossil Record of Birds and its Paleobiological Significance. Chichester, John Wiley \& Sons Ltd.

Nakagawa, S. and Cuthill I. C. 2007. Effect size, confidence interval and statistical significance: a practical guide for biologists. - Biol. Rev. 82: 591-605.

Nemeth, E. and Brumm, H. 2010. Birds and anthropogenic noise: are urban songs adaptive? $-A m$. Nat., 176: $465-475$.

Nonaka, S., Takahashi, R., Enomoto, K., Katada, A. and Unno, T. 1997. Lombard reflex during PAGinduced vocalization in decerebrate cats. - Neurosci. Res. 29: 283-289.

Ord, T. J., Peters, R. A., Clucas, B. and Stamps, J. A. 2007. Lizards speed up visual displays in noisy motion habitats. - Proc. R. Soc. B, 274: 1057-1062.

Osmanski, M. S. and Dooling, R. J. 2009. The effect of altered auditory feedback on control of vocal production in budgerigars (Melopsittacus undulatus). - J. Acoust. Soc. Am. 126: 911-919. 
Pohl, N. U., Slabbekoorn, H., Neubauer, H., Heil, P., Klump, G. M. and Langemann, U. 2013. Why longer song elements are easier to detect: threshold level-duration functions in the great tit and comparison with human data. - J. Comp. Physiol. A 199: 239-252.

Potash, L. M. 1972. Noise-induced changes in calls of the Japanese quail. - Psychon. Sci., 26: 252-254.

Potvin, D. A. and Mulder, R. A. 2013. Immediate, independent adjustment of call pitch and amplitude in response to varying background noise by silvereyes (Zosterops lateralis). - Behav. Eco. 24: 1363-1368.

Prum, R. O., Berv, J. S., Dornburg, A., Field, D. J., Townsend, J. P., Lemmon, E. M. and Lemmon, A. R. 2015. A comprehensive phylogeny of birds (Aves) using targeted next-generation DNA sequencing. Nature 526: 569-573.

Pytte, C. L., Rusch, K. M. and Ficken, M. S. 2003. Regulation of vocal amplitude by the blue-throated hummingbird, Lampornis clemenciae. - Animal Behaviour, 66: 703-710.

Schuster, S., Zollinger, S. A., Lesku, J. A. and Brumm, H. 2012. On the evolution of noise-dependent vocal plasticity in birds. - Biol. Lett. 8: 913-916.

Schwartz, J. J. and Bee, M. A. 2013. Anuran acoustic signal production in noisy environemnts. In: Brumm, H. (ed.) Animal Communication and Noise. Springer-Verlag, Berlin Heidelberg, pp. 91-132.

Shen, J. X. and $\mathrm{Xu}, \mathrm{Z}$. M. 2016. The Lombard effect in male ultrasonic frogs: Regulating antiphonal signal frequency and amplitude in noise. -Sci. Rep. 6.

Sinnott, J. M., Stebbins, W. C. and Moody, D. B. 1975. Regulation of voice amplitude by the monkey. J. Acoust. Soc. Am. 58: 412-414.

Stowe, L. M. and Golob, E. J. 2013. Evidence that the Lombard effect is frequency-specific in humans. J. Acoust. Soc. Am. 134: 640-647.

Templeton, C. N., Zollinger, S. A. and Brumm, H. 2016. Traffic noise drowns out great tit alarm calls. Curr. Biol. 26: R1173-R1174.

Van Summers, W., Pisoni, D. B., Bernacki, R. H., Pedlow, R. I. and Stokes, M. A. 1988. Effects of noise on speech production: Acosutic and perceptual analyses. - J. Acoust. Soc. Am. 84: 917-928.

Wiley, H. 2015. Noise matters - the Evolution of Communication, Cambridge, Harvard University Press.

Zollinger, S. A. and Brumm, H. 2011. The Lombard effect. - Curr. Biol. 21: R614-615.

Zollinger, S. A. and Brumm, H. 2015. Why birds sing loud songs and why they sometimes don't. - Anim. Behav. 105: 289-295.

Zollinger, S. A., Slater, P. J. B., Nemeth, E. and Brumm, H. 2017. Higher songs of city birds may not be an individual response to noise. - Proc. R. Soc. B. 284: 20170602. 


\section{FIGURE LEGENDS}

Figure 1: Typical separation call of a mallard duckling. In this example the call series consists of 7 elements. In our sample the number of elements per series ranged between 6-23.

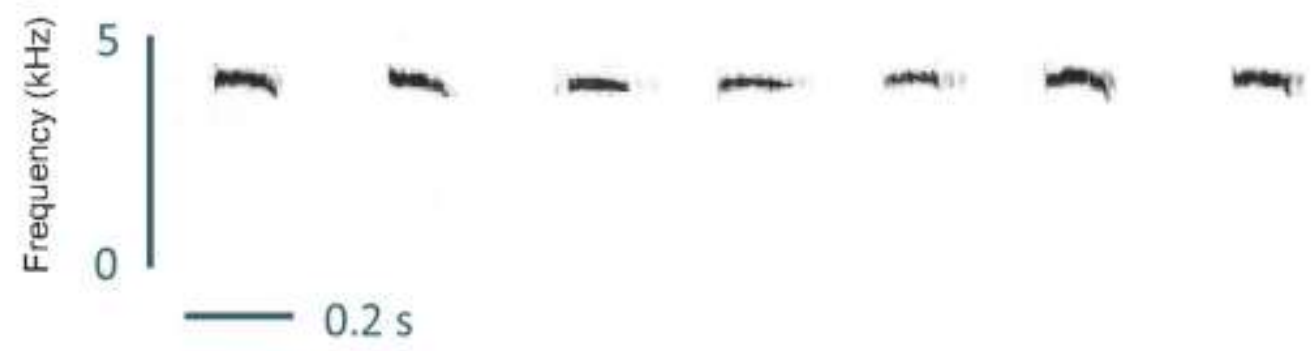


Figure 2: Change in call characteristics as a function of noise level. Data points give the mean estimates of the models with $95 \%$ credible intervals. Call level and call peak frequency varied significantly with the noise treatment, whereas the other variables did not (see Table 1 for details).
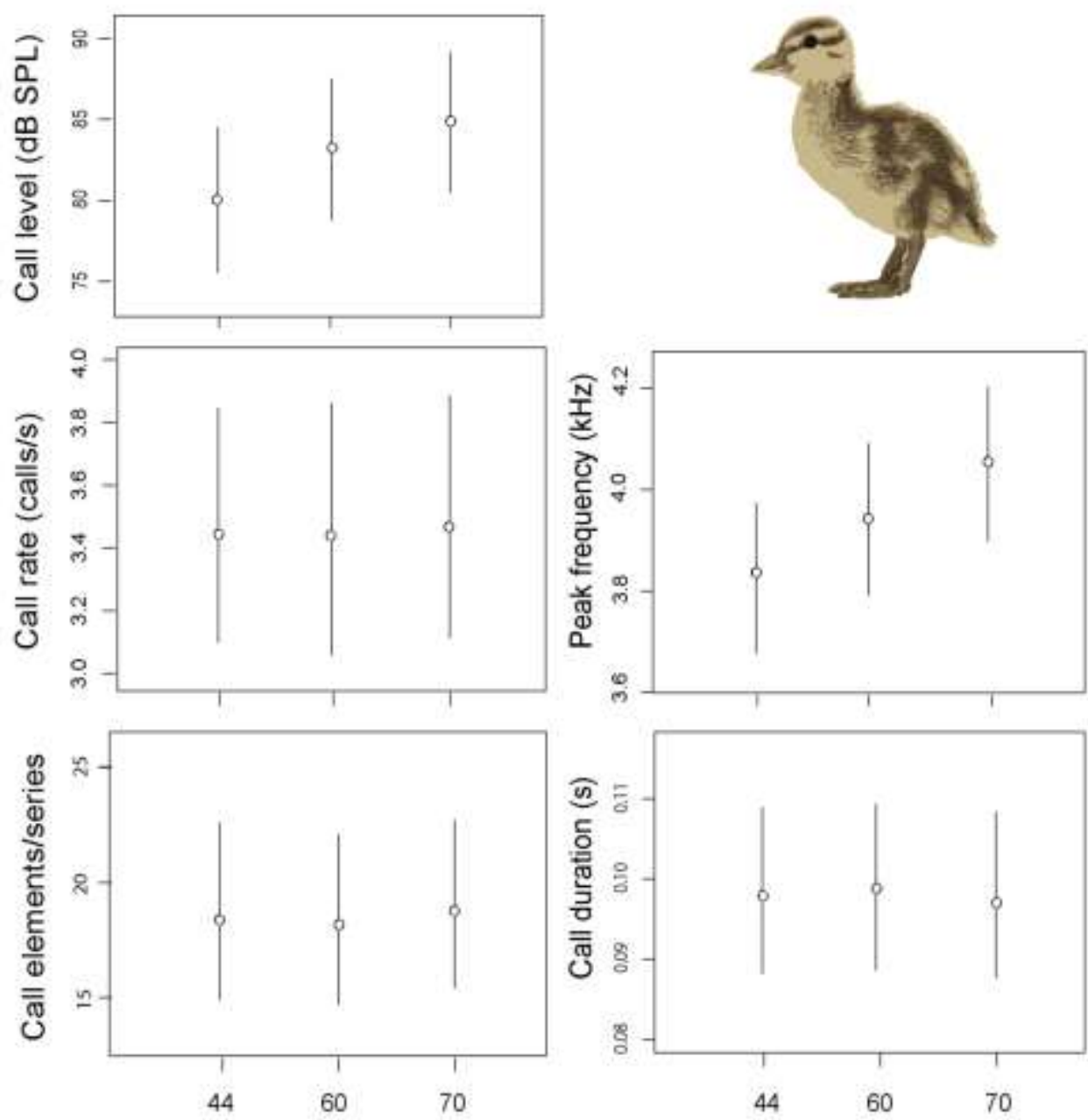

Background noise level (dB) 
Figure 3: The strength of the Lombard effect in mallard ducklings as function of age in mallard ducklings. The Lombard strength is measured as the average increase in call level (in $\mathrm{dB}$ ) between the quiet control and the $70 \mathrm{~dB}(\mathrm{~A}) \mathrm{SPL}$ noise playback.

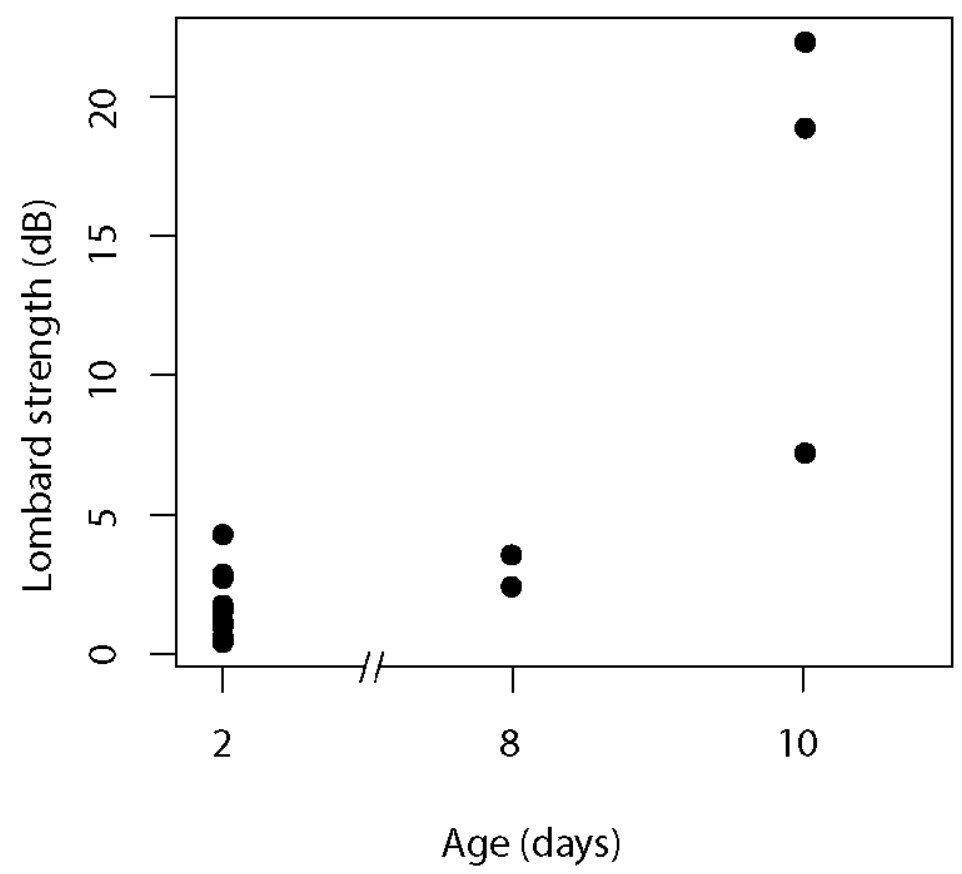

'This article is protected by copyright. All rights reserved.' 
Figure 4: Relation between peak frequency and call level in mallard separations calls. Colours denote different individuals. Across all individuals, the two variables were significantly correlated with each other (see results for details).

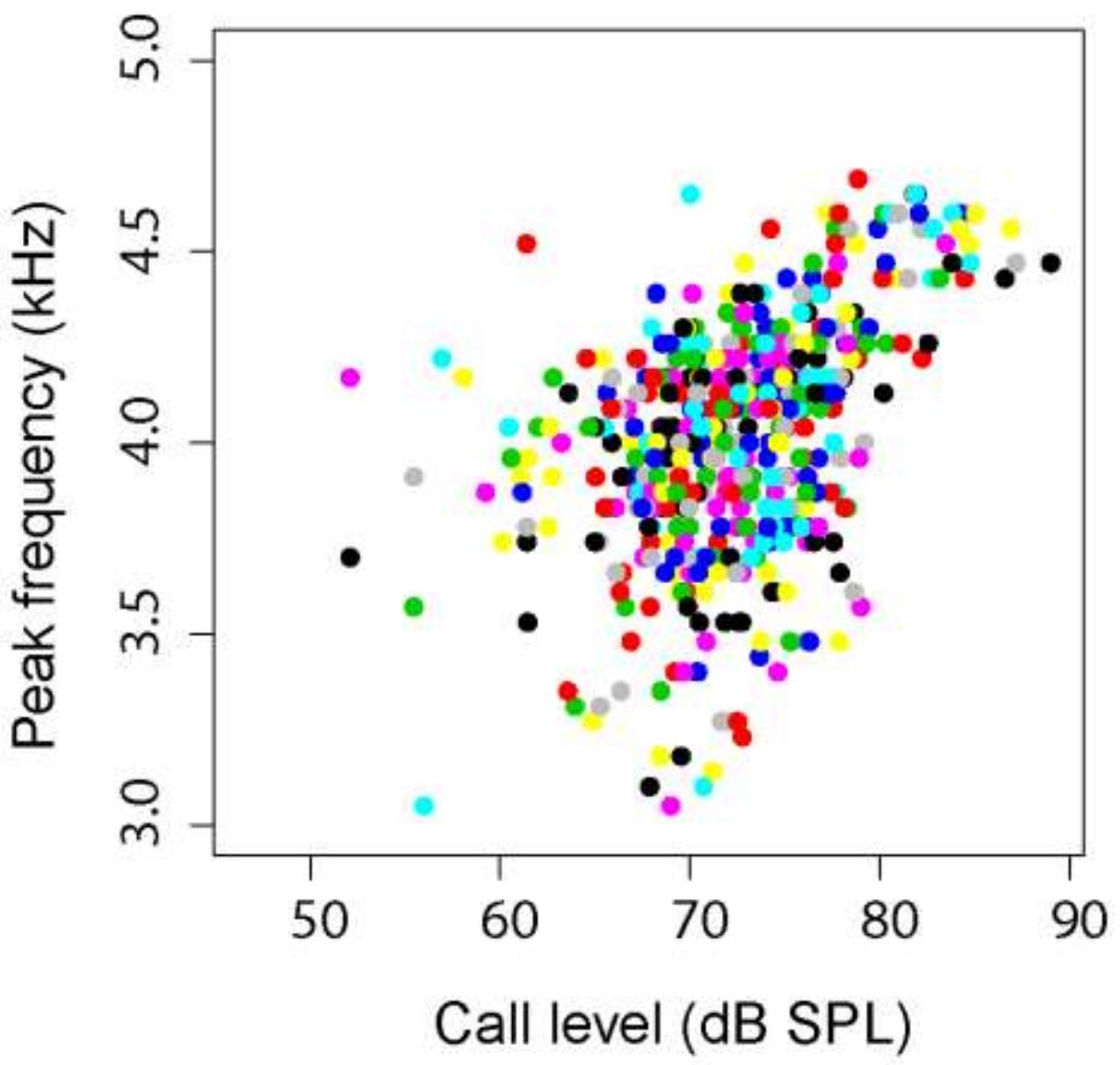

'This article is protected by copyright. All rights reserved.' 
Figure 5: The evolution of the Lombard effect in birds. Plus (+): Lombard effect present, question mark (?): character state not documented. The cladogram is based on Prum et al. (2015).

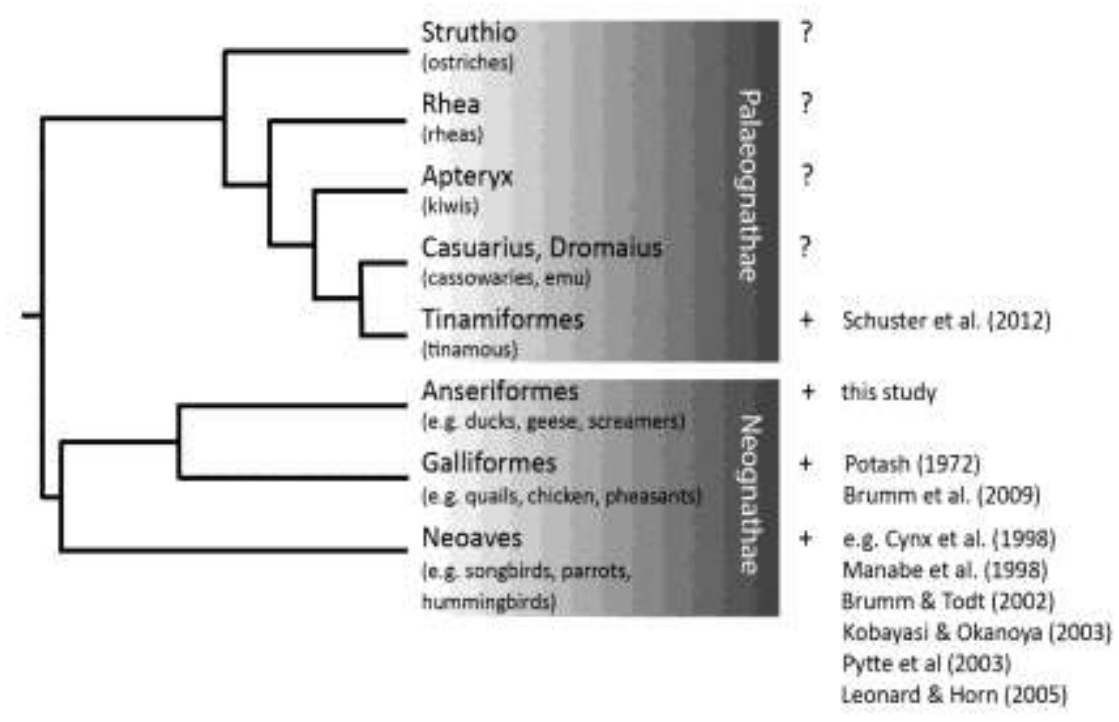




\section{TABLE LEGEND}

Table 1: Outcomes of models testing the effects of three noise treatments on acoustic characteristics of mallard separation calls.

\begin{tabular}{|c|c|c|}
\hline Parameters & Estimate ( $\beta$ ) & $95 \% \mathrm{CrI}$ \\
\hline $\begin{array}{l}\text { Call level (Im) } \\
\text { Fixed effects } \\
\text { (Intercept) } 45 \mathrm{db} \\
60 \mathrm{db} \\
70 \mathrm{db} \\
\text { Random effects } \\
\text { Individual ID }\end{array}$ & $\begin{array}{l}79.72 \\
3.14 \\
4.82 \\
\text { Std. Dev }\left(\boldsymbol{\sigma}^{2}\right) \\
3.05\end{array}$ & $\begin{array}{l}77.36,82.20 \\
0.47,5.89 \\
1.98,7.53\end{array}$ \\
\hline $\begin{array}{l}\text { Peak frequency }(\mathbf{I m}) \\
\text { Fixed effects } \\
\text { (Intercept) } 45 \mathrm{db} \\
60 \mathrm{db} \\
70 \mathrm{db} \\
\text { Random effects } \\
\text { Individual ID }\end{array}$ & $\begin{array}{l}3825.20 \\
117.63 \\
227.41 \\
\text { Std. Dev }\left(\boldsymbol{\sigma}^{2}\right) \\
232\end{array}$ & $\begin{array}{l}3667.39,3972.68 \\
-8.57,248.45 \\
103.83,359.73\end{array}$ \\
\hline $\begin{array}{l}\text { Call rate (glm) } \\
\text { Fixed effects } \\
\text { (Intercept) } 45 \mathrm{db} \\
60 \mathrm{db} \\
70 \mathrm{db} \\
\text { Random effects } \\
\text { Individual ID }\end{array}$ & $\begin{array}{l}1.23 \\
0.009 \\
0.010 \\
\text { Std. Dev }\left(\boldsymbol{\sigma}^{2}\right) \\
0\end{array}$ & $\begin{array}{l}1.11,1.34 \\
-0.15,0.17 \\
-0.15,0.17\end{array}$ \\
\hline $\begin{array}{l}\text { Call duration (Im) } \\
\text { Fixed effects } \\
\text { (Intercept) } 45 \mathrm{db} \\
60 \mathrm{db} \\
70 \mathrm{db} \\
\text { Random effects } \\
\text { Individual ID }\end{array}$ & $\begin{array}{l}0.097 \\
0.0006 \\
-0.001 \\
\text { Std. Dev }\left(\boldsymbol{\sigma}^{2}\right) \\
0.019\end{array}$ & $\begin{array}{l}0.08,0.11 \\
-0.005,0.00 \\
-0.006,0.004\end{array}$ \\
\hline $\begin{array}{l}\text { Number of call elements per series (glm) } \\
\text { Fixed effects } \\
\text { (Intercept) } 45 \mathrm{db} \\
60 \mathrm{db} \\
70 \mathrm{db} \\
\text { Random effects } \\
\text { Individual ID }\end{array}$ & $\begin{array}{l}2.91 \\
-0.02 \\
0.02 \\
\text { Std. Dev }\left(\boldsymbol{\sigma}^{2}\right) \\
0.33\end{array}$ & $\begin{array}{l}2.71,3.12 \\
-0.14,0.18 \\
-0.19,0.15\end{array}$ \\
\hline
\end{tabular}

